\title{
Protée
}

\section{La pelle et le masque}

\section{Les arts dits primitifs sont-il des ready-made?}

\section{Christine Galaverna}

Volume 32, numéro 1, printemps 2004

Mémoire et médiations

URI : https://id.erudit.org/iderudit/011029ar

DOI : https://doi.org/10.7202/011029ar

Aller au sommaire du numéro

\section{Éditeur(s)}

Département des arts et lettres - Université du Québec à Chicoutimi

ISSN

0300-3523 (imprimé)

1708-2307 (numérique)

Découvrir la revue

Citer cet article

Galaverna, C. (2004). La pelle et le masque : les arts dits primitifs sont-il des ready-made? Protée, 32(1), 91-99. https://doi.org/10.7202/011029ar

\section{Résumé de l'article}

Certains effectuent un rapprochement entre arts dits primitifs et ready-made. Séduisante au premier abord par sa simplicité et son économie (elle permettrait de rendre compte, une fois pour toutes, du passage de ce type d'objets de la catégorie des artefacts à celle des oeuvres d'art), cette solution s'avère à l'examen insatisfaisante en ce qu'elle oublie l'importance des conditions de production des objets considérés dans leur fonctionnement ultérieur. Insatisfaisante également en ce qu'elle conduit à ne pas prendre en compte les producteurs des objets dits primitifs. d'utilisation que vous pouvez consulter en ligne.

https://apropos.erudit.org/fr/usagers/politique-dutilisation/ 


\title{
LA PELLE ET LE MASQUE LES ARTS DITS PRIMITIFS SONT-IL DES READY-MADE?
}

\author{
CHRISTINE GALAVERNA
}

\section{Introduction}

Dans un article consacré au futur musée du quai Branly ${ }^{1}$, Jean Bazin et Alban Bensa établissent une comparaison entre ready-made et arts dits primitifs:

Il faut admettre que n'importe quel produit d'un travail humain, d'une activité intentionnelle - tout "artefact", tout ce qui est fait avec un certain art, mais pas forcément "pour l'art» - est susceptible de devenir, dans certaines conditions, une ouvre: Marcel Duchamp nous en a administré une fois pour toutes la démonstration en acte, même si la théorie de l'art n'a pas fini d'en découdre avec la nature énigmatique de cet acte. Il n'est pas indifférent que son intervention inaugurale soit contemporaine de l' invention" des arts dits primitifs. Un masque africain [...] devient une auvre de la même manière qu'un séchoir à bouteille: quand il cesse de servir. ${ }^{2}$

On trouve le même type de rapprochement dans un article de Charlotte Townsend-Gault intitulé explicitement «Ready-made kwakiults?»:

Les ready-made tout comme les plats kwakiults étaient dans leur contexte d'origine des objets courants [...]. Aujourd'bui, les ready-made comme les plats kwakiults sont présentés au public dans un cadre muséal, parce qu'ils ont été les uns et les autres déclassés dans la catégorie des œuvres d'art. ${ }^{3}$

Ce parallèle entre ready-made et arts dits primitifs tente d'apporter une réponse à la question suivante: "Comment un objet, alors que tout laisse penser qu'il n'a pas été composé et fabriqué pour être une œuvre d'art, devient-il une œuvre d'art? " ${ }^{4}$. Il s'agit donc de clarifier le statut des arts dits primitifs à la lueur du ready-made. Car si la valeur patrimoniale de ces objets ne fait aujourd'hui aucun doute, en revanche, ce qu'ils sont demeure problématique. La polémique qui a accompagné l'annonce du projet parisien en témoigne, polémique dont J. Bazin et A. Bensa se font l'écho:

Que va-t-on choisir de nous y montrer? Des documents ou des auvres? De quoi va-t-on nous y parler? D'ethnologie ou d'esthétique? Qu'un tel musée se doive d'être aussi un lieu de recherche, certes! Mais pour quel savoir? 5

Face à cette difficulté de dire ce que sont ces objets que l'on qualifie de primitifs, il n'est pas étonnant que la comparaison avec les ready-made serve à expliquer la présence de ces objets dans le champ artistique. Elle a l'avantage de rendre compte, de manière économique, du passage de l'objet d'une catégorie à une autre, de l'artefact à l'œuvre d'art. L'argument sur lequel se fonde la comparaison est, en effet, celui de la défonctionnalisation: tout comme l'objet constituant le ready-made, l'objet dit primitif devient œuvre d'art à partir du moment où il est coupé de sa fonction. L'économie consiste à ne pas faire reposer le statut d'œuvre d'art sur les qualités artistiques et esthétiques (hypothétiquement) intrinsèques à l'objet, ce qui a l'avantage, notamment, d'éviter les analyses des "arts primitifs" en termes de dévoilement, de révélation (seuls les Occidentaux seraient capables de déceler ces propriétés restées cachées à leurs propres producteurs et utilisateurs).

Cependant, l'analogie est rapidement rabattue sur un rapport de cause à effet: Bazin et Bensa font en effet du 
geste duchampien le préalable à la reconnaissance (à l'invention) des arts dits primitifs. De la même manière, Charlotte Townsend-Gault affirme:

Ce processus [de reclassement] lui-même est tributaire pour une part non négligeable du geste historique de Duchamp, lequel a exercé une influence bien connue sur les classements subséquents d'objets en tant qu'ouvres d'art et sur le développement d'une approche critique à l'égard des critères définissant ce qui est de l'art. ${ }^{6}$

C'est précisément lorsque l'analogie devient rapport de cause à effet qu'elle nous semble suspecte: l'économie n'est plus alors seulement celle d'une définition essentialiste de l'art, mais celle, beaucoup plus problématique, des conditions de production de l'objet (tant d'ailleurs de l'objet dit primitif que du ready-made). L'assimilation pure et simple des arts "primitifs» à des ready-made nous semble méconnaître autant le mode de fonctionnement des ready-made que celui des arts primitifs. Elle met l'accent sur un point de ressemblance en oubliant tout ce qui les éloigne. Les différences entre les deux processus (celui qui fait entrer une pelle dans un musée et celui qui y fait entrer un masque) rendent pourtant l'analogie artificielle et fragile, comme nous nous proposons de le montrer ici, d'abord en revenant sur le geste duchampien, ensuite en reprenant la comparaison de manière approfondie. Cet examen doit nous permettre de comprendre pourquoi une telle comparaison, que nous pouvons d'ores et déjà qulifier de vaine, est faite, de quoi elle est le symptôme.

\section{Le geste duchampien}

Le geste duchampien ${ }^{7}$ est exemplaire en ce qu'il ébranle les critères selon lesquels on juge une œuvre d'art, tant du point de vue de la production que de la diffusion ou encore de la réception.

Lorsque Duchamp expose un porte-bouteille ou une pelle, il importe dans le champ de l'art un objet manufacturé, doté d'une fonction, destiné à remplir un usage, que n'importe qui peut acquérir. En mettant l'accent sur le «tout fait», il met d'abord à mal l'idée selon laquelle l'œuvre est le résultat du travail de l'artiste. L'importance du savoir-faire décroit, voire disparaît:

Un ready-made est un objet tout fait, et déclarer le rapport de l'objet à l'auteur comme une rencontre conduit immédiatement à éliminer le présupposéselon lequel l'auteur a fait l'objet de ses mains. Avec ce présupposé du faire c'est toute la valorisation du métier, tout ce qui, légiférant sur la belle ouvrage, veut que l'objet soit investi d'un savoir-faire, qui bascule dans le refrain en durée, la formule qu'on ressasse, le préjugé idéologique. ${ }^{8}$

Cela ne signifie pas pour autant que l'artiste se retire du processus. Au contraire, sa place demeure centrale. Simplement, au savoir-faire se substitue le choix:

Le mot "art", d'ailleurs, étymologiquement, veut dire faire, tout simplement faire [...]. Qu'est-ce que faire? Faire quelque chose, c'est choisir un tube de bleu, un tube de rouge, en mettre un peu sur sa palette, et toujours choisir la qualité du bleu, la qualité du rouge, et toujours choisir la place sur laquelle on va le mettre sur la toile, c'est toujours choisir. Alors, pour choisir, on peut se servir de tubes de couleur, on peut se servir de pinceaux, mais on peut aussi se servir d'une chose toute faite, qui a été faite, ou mécaniquement, ou par la main d'un autre homme, même, si vous voulez, et se l'approprier, puisque c'est vous qui l'avez choisi. ${ }^{9}$

L'objet accède donc au statut d'œuvre en vertu du choix et de la décision de l'artiste. Le geste artistique est un geste d'appropriation ${ }^{10}$; un geste qui opère un transfert d'une catégorie à l'autre.

En mettant à mal le critère du travail, Duchamp met alors directement en question le critère de l'unicité de l'œuvre (l'œuvre, considérée comme le produit du travail de l'artiste, d'un individu, est quelque chose de rare et d'unique, comme en témoigne l'importance de la signature dans l'authentification d'une œuvre):

Un des aspects du ready-made est qu'il n'a rien d'unique [...]. La réplique d'un ready-made transmet le même message; en fait presque tous les ready-made existants aujourd'hui ne sont pas des originaux au sens reçu du terme. ${ }^{11}$

La valeur artistique de l'œuvre ne se situe donc pas dans l'objet, mais dans le choix qui l'a extrait de son contexte «normal» de fonctionnement. Elle ne se situe pas dans l'objet, c'est-à-dire qu'elle ne découle pas des propriétés de l'objet choisi. Duchamp met l'accent sur ce point. La banalité même de l'objet est censée désamorcer le goût esthétique:

Il est un point que je veux établir très clairement, c'est que le choix de ces ready-made ne me fut jamais dicté par quelque 
délectation esthétique. Ce choix était fondé sur une réaction d'indifférence visuelle, assortie au même moment à une absence totale de bon ou de mauvais goût [...] en fait une anesthésie complète. ${ }^{12}$

Le ready-made tente ainsi de désamorcer ce sur quoi se fonde la réception de l'œuvre: l'expérience de propriétés esthétiques censées appartenir en propre à l'objet.

Le geste duchampien s'inscrit donc dans un monde de l'art précis (en l'occurrence le monde de la peinture si l'on suit sur ce point Thierry de Duve ${ }^{13}$ ). Il se pose en réaction face à l'impossibilité, dans un monde industriel où la répétition et la série sont reines, de faire de la peinture sur le mode classique. Ce geste s'institue (ou peut se comprendre) comme un nouveau paradigme artistique ${ }^{14}$.

Ce geste étant posé, qu'en est-il de la comparaison avec les arts dits primitifs?

\section{Le statut initial des objets: des artefacts}

Nous l'avons dit en introduction, l'argument principal sur lequel repose la comparaison est celui de la défonctionnalisation de l'objet (qui à son tour repose sur le présupposé de la gratuité de l'art, de l'œuvre ne valant que par ellemême) : l'objet, dans le musée, ne sert plus. Cette défonctionnalisation marque le passage de l'objet considéré de la classe des artefacts à celle des œuvres d'art. Et effectivement, le masque africain ou le plat kwakiult sont des artefacts dans la mesure où ils ne sont pas produits initialement pour fonctionner comme ouvres d'art ${ }^{15}$. Cependant, s'agit-il du même type d'artefacts qu'une pelle ou un portebouteille? La question peut sembler étrange. En effet, qu'entend-on par "du même type"? Vise-t-on la fonction initiale de l'objet? Certes, un masque n'a pas la même fonction qu'une pelle. Mais la pelle à son tour n'a pas la même fonction qu'un porte-bouteille ou un urinoir. Ce n'est donc pas tant la fonction qui est visée par cette question que le mode de production de l'objet: là se situe une différence, qui n'a rien d'anecdotique, entre readymade et arts dits primitifs.

Les objets constituant les ready-made sont des objets produits en série, que rien ne distingue donc a priori des objets de la même série. Les objets dits primitifs relèvent d'une tout autre forme de fabrication, qui ressort de l'artisanat. Et dans le cas d'objets culturels, tels un masque ou un plat cérémoniel (pour reprendre les exemples développés dans les deux articles qui retiennent ici notre attention), cette fabrication est soumise, en outre, à des contraintes d'ordres rituel et matériel ${ }^{16}$. Des gestes rituels peuvent être effectués dès le moment de la production, ce qui implique non seulement un savoir-faire technique de la part des personnes engagées dans le processus de fabrication de l'objet, mais également, pour le ou les producteurs, un statut particulier dans la communauté (par exemple, un statut élevé dans la hiérarchie des initiés de la société au sein de laquelle ou pour laquelle le masque est fabriqué). Il ne s'agit donc pas simplement de produire un objet dont la forme est adaptée à la fonction, et dont les étapes de fabrication peuvent être déléguées à d'autres, comme c'est le cas pour un objet manufacturé. De plus, cette mixité de contraintes matérielles et rituelles peut entraîner une polysémie propre à ce type d'objets, comme le souligne Charlotte Townsend-Gault à propos des plats kwakiults:

En raison du lien étroit qui existe entre la nourriture et les animaux, ou la quête du gibier, et entre la nourriture et les cérémonies associées à la confirmation du statut des membres du groupe, la catégorie des plats cérémoniels est représentative d'un ensemble complexe d'idées et de pratique. Pour les autochtones, ces objets ne sont réductibles ni à l'usage auquel ils sont destinés ni à leur signification cosmogonique. Ils sont alternativement des objets fonctionnels et des représentations spirituelles, et ils ont dans l'une et l'autre fonction une signification culturelle particulière. Ils sont, sur les plans matériels et conceptuels, ambigus. ${ }^{17}$

Peut-on dire d'une pelle qu'elle est matériellement et conceptuellement ambiguë? Et pourtant, le rapprochement est fait entre la pelle et le plat: «Ce que le récipient et la pelle ont en commun, c'est que les deux objets peuvent et doivent se prêter à un système de classement multiple» ${ }^{18}$. C'est entendu. Mais initialement, la pelle et le plat n'ont assurément pas la même charge symbolique. S'ils peuvent tous deux être considérés comme des artefacts, puisqu'ils sont dotés d'une fonction et d'un usage, la valeur sacrée attribuée au plat (comme au masque) en fait un objet bien différent de la pelle.

Artefacts, donc, puisqu'ils remplissent une fonction précise et qu'ils sont dotés d'une valeur d'usage; masque africain et plat kwakiult ne le sont pourtant pas au même titre que la pelle ou le porte-bouteille. La question de la 
fonctionnalité de l'objet (et celle de son usage) ne se pose pas de la même façon pour le masque et pour la pelle: une croix chrétienne peut-elle fonctionner véritablement comme ready-made? C'est peu probable, du fait de la charge symbolique religieuse qu'elle véhicule (le désamorçage effectué par Duchamp à propos de l'émotion serait dans ce cas précis fortement compromis). Certes, on pourrait objecter que le masque, notamment, joue dans l'article de Bazin et Bensa un rôle paradigmatique. Il signifie l'ensemble de la catégorie "arts primitifs", le masque étant ce qui le plus souvent est associé à ce type d'art. Par là même, par ce rôle paradigmatique, on pourrait substituer n'importe quel objet au masque. Mais c'est précisément là que se situe le problème: les objets dits primitifs présentés dans les musées occidentaux ne subissent aucune discrimination: tous sont exposés sur le même plan, qu'il s'agisse d'un objet de la vie quotidienne, comme une cuiller ou un plat, ou d'un objet chargé de valeurs symboliques, tel un masque. Ce nivellement a pour conséquence de fondre en une même catégorie des objets qui, en Occident, sont tenus soigneusement séparés. Dit-on d'une cuiller, produite en Occident et présentée dans un musée, qu'elle est ready-made? Non, parce qu'elle n'est pas considérée comme une œuvre d'art, mais comme un objet d'art.

Si donc la fonction à laquelle ils sont destinés et leur mode de fabrication font du masque africain ou du plat cérémoniel des artefacts en un sens différent de celui de la pelle ou du porte-bouteille, sa négation, à l'origine de leur insertion dans le musée, prend-elle conséquemment un sens différent? Peut-elle être considérée comme un processus équivalent?

\section{Deux modes de défonctionnalisation}

Le terme de défonctionnalisation rend compte de l'extraction de l'objet de son contexte initial, de la négation de son utilité (il ne sert plus, il n'est plus utilisé). Cependant, si la pelle ne sert plus à déneiger, il n'en demeure pas moins que la référence à son usage initial est un des éléments du fonctionnement de In Advance of a Broken Arm. L'anesthésie requise par Duchamp découle précisément du fait que l'objet choisi est un objet de la vie courante, dont la forme sert la fonction et ne prend sens que par rapport à elle: la forme de l'objet dit sa fonction, inévitablement ${ }^{19}$. Défonctionnaliser ne signifie donc pas nier la fonction, mais la transférer en un autre contexte: elle n'est plus entée sur un usage donné, mais tient lieu de référence sémantique du fonctionnement artistique de l'objet.

Ce processus se retrouve lors du passage des arts primitifs de la catégorie des artefacts à celle des œuvres d'art: tout comme la pelle de In Advance of a Broken Arm ne servira plus à déneiger, le masque, une fois qu'il a intégré l'institution muséale (qu'elle ait pour mandat de les présenter comme des documents ou comme des œuvres d'art ${ }^{20}$ ) ne sera plus porté. Mais tout comme dans le cas de l'objet constituant le ready-made, la fonction initiale de l'objet demeure présente. Elle transparaît d'abord dans l'identification des objets: "masque", "plat", "cuiller", etc. On trouve là une autre différence entre ready-made et arts primitifs. La pelle n'est pas exposée en tant que pelle, mais en tant que In Advance of a Broken Arm, c'est-à-dire qu'elle subit une transformation par la titulation: le titre fournit une identité nouvelle à l'objet, il participe de sa nouvelle désignation en tant qu'œuvre d'art. Le masque, lui, est exposé en tant que masque. Il n'a pas de titre à proprement parler, mais est désigné par son identité fonctionnelle (la désignation de l'objet est tautologique).

La fonction de l'objet joue également un rôle dans l'authentification (donc dans l'évaluation de la valeur marchande) de l'objet: "What dealers, collectors, and art historians call "authentique" Primitive or Traditional Art is a piece 1) made by a member of a small-scale society, 2) in the society's traditional style, and 3) intended for a traditional social or religious function " 21 . Un objet qui n'aura pas servi aura donc moins de valeur qu'un objet qui aura servi, voire sera considéré comme une copie ou un faux; la référence à la fonction peut conduire alors à établir une cartographie de l'authenticité, ainsi que le fait Frank Willett:

Les cuvres les plus manifestement authentiques, sur lesquelles tout le monde s'accorderait, sont celles qui ont été faites par un Africain pour l'usage par son propre peuple et qui ont été utilisées ainsi. Cependant, cette catégorie peut être subdivisée, parce que la pièce ainsi faite et utilisée peut être d'une qualité esthétique supérieure, moyenne ou inférieure. Un peu plus bas sur l'échelle se place une cuvre faite par un Africain, pour usage par son propre peuple, mais achetée avant usage par un étranger (expatriate). Vient ensuite une sculpture faite par un Africain, dans le style traditionnel, sur commande d'un étranger. Ensuite une sculpture faite par un Africain, dans une pauvre imitation du style traditionnel de son propre peuple, pour la vente à un étranger. Faite par un Africain, 
dans le style d'un peuple africain différent (bien qu'elle puisse être bien faite), pour la vente à un étranger. Finalement, nous avons les oeuvres faites par un étranger, c'est-à-dire un non-Africain, pour la vente à d'autres non-africains, mais se faisant passer pour africaines. Ces dernières, à l'autre extrémité du continuum, sont des faux indiscutables. ${ }^{22}$

La référence faite à la fonction ne joue cependant pas le même rôle que dans le cas du ready-made: dans ce dernier cas, elle est l'élément du fonctionnement artistique de l'objet; dans le cas d'un masque, elle est le préalable à sa désignation en tant qu'œuvre d'art et donc à son fonctionnement artistique ${ }^{23}$. Le rôle attribué à la fonction differe de celui qu'elle peut avoir dans le ready-made. Cela nous conduit à examiner de plus près ce processus de défonctionnalisation sur lequel se fonde la comparaison qui nous occupe. Il nous semble que cette défonctionnalisation n'a pas le même sens dans un cas et dans l'autre. En effet, la pelle de Duchamp n'a jamais été et «ne sera jamais utilisée, tordue, mangée par la rouille ou gagnée par l'obsolescence» 24 . Au contraire, le masque, pour être une authentique œuvre d'art (pour fonctionner comme œuvre d'art), doit avoir été utilisé. Autrement dit, dans le premier cas, il y a neutralisation initiale de la fonction, dans le sens où il y a absence d'usage (l'objet est privé de son usage). Dans le second cas, il y a neutralisation de l'usage.

Pourquoi mettre l'accent sur cette distinction entre fonction et usage? Ce qui importe dans le cas du readymade, c'est la référence faite à la fonction et non à l'usage, nous l'avons vu. Que la pelle ait été utilisée n'est pas un élément du fonctionnement du ready-made. L'objet est plutôt pris comme matériau, c'est-à-dire comme ce qui va constituer le point de départ de l'œuvre, ce qui va être modifié par l'artiste (la comparaison qu'effectue Marcel Duchamp avec la peinture nous semble aller, pour une part, dans ce sens: la pelle se tient à la même place, dans le processus, que les tubes de peinture). Dans le cas du masque, il en va tout autrement: son utilisation est primordiale dans la reconnaissance de son authenticité, et il est exposé en tant que masque ayant un jour été porté au cours d'une cérémonie. Il n'est pas matériau d'un acte artistique $^{25}$, contrairement à la pelle. Cette différence nous amène alors à considérer la question de la décision à l'origine de l'insertion du masque dans le musée, cette question étant l'autre élément sur lequel repose la comparaison entre ready-made et arts dits primitifs. L'analogie est rendue possible par le fait que, dans les deux cas considérés, la décision revient aux acteurs du monde de l'art: le ready-made interroge le fonctionnement institutionnel du monde de l'art en en brouillant les frontières admises. Mais il ne peut le faire qu'à partir du moment où le geste iconoclaste de Duchamp est relayé par l'institution, par le monde de l'art dans son ensemble. De la même manière, l'apparition des objets africains (et plus généralement des objets provenant de sphères extra-occidentales) découle d'une décision provenant du monde de l'art: ce sont des objets qui ne sont pas au départ des œuvres d'art (tout le discours ethnologique tend à le montrer), qui ne répondent pas à ce que l'on admet comme étant un monde de l'art. Ils sont extraits de leur contexte initial, tout comme la pelle, et intégrés dans un nouveau contexte.

\section{Décision artistique et décision institutionnelle}

Nous l'avons vu, le choix est à l'origine du ready-made. Ce choix est effectué par l'artiste Marcel Duchamp qui le désigne comme un faire: l'élection de l'objet constitue donc l'acte artistique en tant que tel. Le geste amorcé par l'artiste est ensuite relayé par les autres acteurs du monde de l'art dans lequel le ready-made s'inscrit. Même si le ready-made se présente comme une contestation des règles du monde de l'art, même s'il est à l'origine l'objet d'un rejet, il ne prend sens et n'est réalisable qu'au sein de ce monde de l'art (ou d'un certain état de ce monde de l'art). En ce sens, le geste artistique est également le fruit d'une décision institutionnelle (voir le geste reconnu, être accepté comme ayant une cohérence et une pertinence artistique). L'artiste demeure cependant propriétaire de son geste, dans une certaine mesure, puisque c'est lui qui décide du nombre de répliques des ready-made, qui en réglemente la production:

Très tôt je me rendis compte du danger qu'il pourrait y avoir à resservir sans discrimination cette forme d'expression et je décidai de limiter la production des ready-made à un petit nombre chaque année. Je m'avisai à cette époque que, pour le spectateur plus encore que pour l'artiste, l'art est une drogue à accoutumance et je voulais protéger mes ready-made contre une contamination de ce genre. 26

Le ready-made s'inscrit donc dans un monde de l'art constitué. 
Il en va tout autrement des arts dits primitifs: la décision qui fait d'eux des œuvres d'art n'est pas un acte artistique, même si les artistes du début du XXe siècle sont pour beaucoup dans cette décision. La décision qui est à l'origine de l'intégration de ces objets dans le champ artistique est indépendante des conditions de production de l'objet; elle s'effectue a posteriori. L'histoire de leur réception le montre: d'abord objets de curiosité, puis objets ethnologiques, les objets provenant des sociétés non occidentales accèdent au rang d'œuvres d'art dans le courant du XXe siècle. En d'autres termes, dans le cas du ready-made, l'intégration d'un objet usuel au champ artistique est le résultat d'un acte artistique; dans le cas des arts dits primitifs, elle résulte d'un acte institutionnel (qui s'effectue, de surcroît, dans une autre aire culturelle que celle de leur production ${ }^{27}$ ).

La conséquence de cette décision est d'accorder, par ricochet, le statut d'artistes aux producteurs de ces objets et d'opérer parallèlement une requalification a posteriori de ces objets: ils sont perçus comme étant dès le départ des œuvres d'art, même s'ils sont dotés d'une fonction et d'un usage. A contrario, jamais le statut d'artiste n'a été accordé au producteur de la pelle (si tant est même que l'on puisse déterminer qui a produit la pelle dans le cadre d'une production industrialisée). Dans le cas du ready-made, les deux sphères (la sphère de production et de fonctionnement de l'objet et celle de son fonctionnement artistique) sont indépendantes l'une de l'autre. La référence au caractère non esthétique du ready-made, à la place initiale de l'objet transporté dans le musée, est, comme on a pu le voir, le moteur de l'œuvre. Mais l'exposition de la pelle dans le musée ne modifie en rien la sphère de production des pelles en général. Dans le cas des objets extra-occidentaux, au contraire, les deux sphères se mêlent étroitement: l'acceptation de ces objets dans le musée modifie par contrecoup le regard que l'on porte sur leurs producteurs et modifie également les conditions de production de ces objets. Nombreux sont les exemples qui font état d'une transformation de la production en vue de satisfaire les attentes et les exigences des collectionneurs occidentaux. Ainsi, tout masque sera désormais considéré comme une œuvre d'art. Bonne ou mauvaise; faux, copie ou authentique, c'est un autre problème. Plus exactement, c'est parce que le masque est classé dans la catégorie "œuvre d'art» que l'on pourra parler de faux ou de copie. En revanche, les pelles ne sont pas considérées, dans leur ensemble, comme des œuvres d'art, parce que Duchamp a commis In Advance of a Broken Arm; elles ne seront pas plus considérées comme des faux ou des copies de cette même œuvre ${ }^{28}$. L'analogie effectuée tant par Bensa et Bazin que par Townsend-Gault se révèle donc, à l'analyse, non pertinente pour rendre compte de l'intégration des objets provenant des sociétés extraoccidentales dans le champ artistique. L'argument de la défonctionnalisation repose sur un oubli paradoxal: celui du geste à l'origine du ready-made. On ne retient que le fait qu'un objet usuel devient une ouvre d'art, mais pas le processus par lequel cet objet devient œuvre, qui est un processus artistique. De quoi un tel oubli est-il le symptôme?

\section{Les arts dits primitifs: des œuvres sans artistes}

Une première hypothèse serait de voir, dans cet oubli, un refus du geste artistique de Duchamp pour n'en retenir que la décision d'ordre institutionnel. Étant donné la postérité du geste, tant du point de vue de la production artistique que des analyses critiques qui lui accordent une valeur inaugurale, cela est peu probable: les ready-made sont définitivement inscrits dans l'histoire de l'art occidental. Par conséquent, si l'on conserve à l'esprit la dimension artistique du geste duchampien, on en arrive à une conclusion hautement problématique: le commissaire d'exposition, qui choisit les artefacts à exposer, qui décide lesquels sont dignes de figurer dans une exposition d'ordre artistique, serait lui-même artiste ${ }^{29}$. On aura quelque hésitation face à une telle conclusion... Il n'en demeure pas moins que, dans le cas des arts dits primitifs, la décision institutionnelle, c'est-à-dire la décision émanant des acteurs de la diffusion (conservateurs, commissaires d'exposition, marchands, etc.), est primordiale dans la reconnaissance du statut artistique des artefacts considérés 30 .

Et la comparaison entre les ready-made et les arts dits primitifs va dans le sens de cette primauté de la diffusion sur la production. En effet, affirmer que le masque et la pelle peuvent être considérés comme art revient à comparer le producteur de la pelle et le producteur du masque. On met sur un même plan le producteur de masque et le producteur de pelle, le producteur d'un objet à teneur religieuse ${ }^{31}$, dont la fabrication s'insère dans un réseau de contraintes rituelles autant que matérielles, et le producteur d'un objet manufacturé en série.

Cette assimilation, sous-entendue par la comparaison avec les ready-made, rend compte de l'ambiguïté rattachée 
à l'appréhension des objets relevant de la catégorie "arts primitifs»: œuvres d'art, certes, mais œuvres sans artistes. Si l'on accepte d'accorder à ces objets le statut d'œuvres d'art, l'attribution du statut d'artiste à leurs producteurs est plus problématique. Longtemps le discours occidental s'est satisfait de l'anonymat des arts dits primitifs: considérés comme des produits de la collectivité, ces objets n'étaient pas censés répondre à une production individualisée. Longtemps, et même encore maintenant, comme le souligne Sally Price:

Quelle que soit son origine, l'anonymat joue un rôle important dans l'image de "l'art primitif» dans le monde occidental. Un marchand parisien avec qui j'ai discuté de ce phénomène l'a bien résumé en déclarant: "Si l'artiste n'est pas anonyme, l'art n'est pas primitif». 32

L'auteure souligne également le rôle que joue l'anonymat au sein du marché de l'art: introduits la plupart du temps en Occident par l'intermédiaire des marchands et collectionneurs, plutôt que par celui des ethnologues et historiens de l'art, ces objets se trouvent dépossédés de leur identité pour en acquérir une nouvelle en Occident:

Ce remplacement d'un passeport étranger par une carte d'identité familière sert à faciliter l'introduction de l'objet dans un système qui tourne autour de l'esthétique occidentale et de sommes d'argent importantes. En abandonnant ses "signatures», pour aboutir aux "pedigrees» occidentaux, on effectue un transfert de responsabilité en ce qui concerne sa paternité artistique. 33

Ce transfert de responsabilité va de pair avec l'idée selon laquelle l'artiste "primitif" n'a pas conscience de faire de l'art et n'est donc pas responsable du résultat. Seul l'Occident est en mesure de révéler le caractère artistique de l'objet ${ }^{34}$.

Et c'est bien ce «transfert de paternité artistique» qui est ici en jeu dans la comparaison effectuée entre les arts dits primitifs et les ready-made: en assimilant le processus d'intégration des objets provenant des sociétés extra-occidentales à celui qui fait entrer une pelle dans un musée, on nie purement et simplement l'importance du contexte de production et du contexte initial de fonctionnement dans leur appréhension en tant qu'œuvres d'art: ces objets ne commencent leur carrière artistique qu'à partir du moment où les Occidentaux les considèrent comme des œuvres. Et si l'on a pu trouver saugrenue l'hypothèse qui conduirait à faire des commissaires d'exposition des artistes, on n'en est pourtant pas très loin lorsqu'on examine le rôle que joue le pedigree dans la détermination de la valeur artistique de l'objet. Sally Price rapporte les propos d'un marchand parisien: «Le pedigree, ça vaut la signature» 35 . Autrement dit, le possesseur de l'objet, le collectionneur occidental, se substitue au producteur de l'objet: peu importe qui a fabriqué l'objet; seul compte le regard du connaisseur occidental. Et considérer un masque africain ou un plat cérémoniel kwakiult comme des ready-made s'inscrit, volontairement ou involontairement, dans cette «idéologie» d'un art anonyme.

\section{Conclusion}

Loin de résoudre les ambiguïtés que produit l'intégration des objets provenant des sociétés extra-occidentales au champ de l'art, la comparaison avec le "processus readymade» ne fait donc que les reconduire: déclassement de la catégorie «artefact» à la catégorie «œuvre d'art» fondé sur la défonctionnalisation, et pourtant référence à l'usage initial de l'objet pour la détermination de sa valeur artistique; œuvres sans artistes, et pourtant affirmation d'une valeur artistique initiale qui se dévoilerait sous le regard du connaisseur occidental; oubli de la dimension artistique du geste duchampien dans le parallèle effectué, et pourtant instauration d'un lien de cause à effet entre la présence de la pelle dans le musée d'art et celle des arts dits primitifs. Ce que cette comparaison nous dit, au contraire, c'est la primauté du regard occidental, et plus particulièrement celui des acteurs de la diffusion, dans la détermination de la valeur artistique (et donc marchande) des œuvres considérées. La conséquence est grave: assimiler ces objets à des ready-made revient purement et simplement à les annexer à l'histoire de l'art occidentale et interdit à terme la possibilité même d'une histoire de l'art proprement non occidental. En effet, comment des "œuvres sans artistes" pourraientelles faire l'objet d'une histoire de l'art quand celle-ci est considérée comme

[...] mosaïque de contributions faites par des individus dont les noms sont connus, dont les aeuvres peuvent être distinguées, et dont les vies personnelles et le rapport qu'ils ont eu avec leur époque méritent d'attirer notre attention? 36 


\section{NoTES}

1. Le futur musée du quai Branly, dont l'ouverture est prévue pour 2004, doit accueillir les collections d'ethnologie de l'actuel Musée de l'homme et les collections du Musée des arts africains et océaniens (Paris) qui a fermé ses portes début 2003. Le quai Branly se veut un musée des cultures non européennes (Afrique, Océanie, Amérique indienne, Insulinde et régions de l'Asie non représentées au musée Guimet).

2. J. Bazin et A. Bensa, "À propos d'un musée flou », Le Monde, 19 avril 2000.

3. C. Townsend-Gault, «Ready-made kwakiults?», dans J. Bradley et L. Johnstone (sous la dir. de), Réfractions. Trajets de l'art contemporain au Canada, Montréal/Bruxelles, Éd. Artextes/La Lettre volée, 1998, p. 158168, ici p. 163. Les Kwakiults sont une population autochtone de la côte nord du Pacifique. «Les plats des Kwakiults sont faits généralement de bois d'aulne; ce sont soit des pièces de bois évidées, soit des planches rainurées et courbées formant un récipient rectangulaire. La taille des plats cérémoniels peut varier considérablement, allant de quelques centimètres à plus de deux mètres, ces dernières dimensions correspondant intentionnellement à celles d'un petit canoë. Ils empruntent leurs formes aux corps des animaux et des humains ou ils représentent des esprits de la cosmogonie kwakiult. Le plat proprement dit est l'estomac ou le dos évidé de ces figures. Ces plats ne sont qu'un des ensembles d'objets propres aux cultures de la côte nord du Pacifique - boîtes à provisions, couvertures, bâtons d'orateur, hochets, masques -, qui sont autant de parties inextricables d'un tout comprenant aussi des éléments immatériels - titres cérémoniels, chants, histoires familiales, mythes, danses, privilèges de toutes sortes». Ibid., p. 159-160.

4. J. Bazin et A. Bensa, art. cit.

5. Ibid. D'une manière plus large, ce questionnement sur le statut des objets constituant la catégorie " arts primitifs» se trouve renforcé notamment par les revendications des Autochtones d'Amérique concernant leur patrimoine disséminé dans les musées occidentaux : ces objets sont avant tout pour eux des objets de culte de peuples vivants. L'argument rejoint ici l'argument de l'ethnologie: c'est également au nom de cette fonction originelle de l'objet que le fonctionnement artistique est considéré comme étant non pertinent par nombre d'ethnologues. Cependant, cette proximité n'est qu'apparente. En effet, ce que les revendications des populations autochtones visent, c'est le fonctionnement muséal de l'objet : «Le public a-t-il le droit de tout savoir, de voir les choses qu'une autre culture considère comme trop sacrées pour être exposées, de posséder le patrimoine culturel et spirituel d'un autre peuple?» (R. Hill, «Un dépôt sacré: les obligations culturelles des musées envers les Autochtones ", Muse, vol. VI, $\mathrm{n}^{\circ} 3$, octobre 1998, p. 34-36; ici p. 36). À ce titre, l'approche ethnologique est tout autant visée que l'approche artistique dans la mesure où le fonctionnement ethnologique de l'objet passe par l'exposition.

6. C. Townsend-Gault, art. cit., p. 164

7. Il ne s'agit pas ici de donner une analyse (une de plus) des ready-made. Ce qui nous intéresse est le rapport établi entre ce processus et celui de l'intégration des objets extra-occidentaux au champ del'art. C'est pourquoi nous ne nous attarderons que sur les points qui permettent à l'analogie de fonctionner, et non sur l'ensemble des questions que soulève le "cas readymade".

8. T. de Duve, Résonances du readymade. Duchamp entre avant-garde et tradition, Nîmes, Éd. Jacqueline Chambon, 1989, p. 21-23.

9. M. Duchamp, Entretiens inédits avec Georges Charbonnier, RTF, 1961, cité par T. de Duve, op. cit., p. 142-143.

10. La titulation (le fait de donner un titre à) des ready-made en est le signe le plus évident: Duchamp ne se contente pas d'exposer une pelle en la nommant "pelle», mais en lui adjoignant la phrase: "In Advance of a Broken Arm».

11. M. Duchamp, Duchampdusigne, Paris, Flammarion/Champs, (1975)
1994, p. 192. La réplique n'en demeure pas moins sous contrôle de l'artiste: seul Duchamp peut décider et approuver (authentifier) une réplique d'un ready-made. Le geste duchampien n'implique donc pas que n'importe qui puisse décider de faire un ready-made.

12. Ibid., p. 191. Nous disons "censée» car, si les ready-made peuvent effectivement ne pas susciter de délectation esthétique (encore que certains les louent aujourd'hui pour leurs qualités formelles), ils n'en suscitent pas moins, au départ, le rejet et donc une manifestation de dégoût.

13. «De toute évidence le ready-made est, entre autres choses, l'enregistrement de son abandon de la peinture, la manière dont ce dernier se trouve porté au registre et, par là-même, rendu signifiant. Il signifie à la peinture qu'elle est morte et le lui fait signifier en n'étant plus peinture. Ne fût-ce que pour cette raison, les ready-made appartiennent paradoxalement à l'histoire de la peinture et non, par exemple, à celle de la sculpture». T. de Duve, op. cit., p. 132

14. Sur l'analyse du ready-made comme paradigme, $c f$. T. de Duve, op. cit., p. 7-57.

15. C'est précisément sur cette question que reposent les critiques envers une approche purement esthétique des objets: nombre d'ethnologues considèrent que faire d'un masque une œuvre d'art, c'est lui ôter toute signification en lui assignant un statut par trop éloigné de son statut initial le masque est un objet fonctionnel et oublier cela constituerait un non-sens du point de vue de l'ethnologie. Mais c'est oublier un peu vite que, y compris dans le musée d'ethnologie, l'objet est coupé de sa fonction: l'ethnologie entretient en effet l'illusion d'un accès direct à l'objet et à son contexte d'usage, ce qui conduit certains, tel L. Stéphan, à assimiler contexte d'usage et contexte ethnologique («La sculpture africaine. Essai d'esthétique comparée", L'Art africain, Paris, Citadelles \& Mazenod, 1988). Mais le discours ethnologique est construction, comme n'importe quel discours "scientifique», et le masque ne fonctionne pas plus en tant que masque dans le musée d'ethnologie que dans le musée d'art. Bazin et Bensa soulignent très bien cette illusion de l'ethnologie et de la muséographie qui lui est rattachée: «Une société, si “primitive” et “sans histoire” qu’elle puisse paraitre, n'est jamais un univers clos: c'est plutôt le regard de l'ethnographe qui tend à l'isoler pour mieux la connaître, à la circonscrire pour mieux la décrire. Dans ce travail de sélection et de classement, la muséographie voudrait asséner des preuves : les objets désignent les ethnies auxquelles on les identifie. Il y a des Dogons puisqu'il y a des masques dogons. L'effet d'authenticité est d'autant mieux assuré que ce raisonnement circulaire construit des monades en forme de vitrines». J. Bazin et A. Bensa, art. cit.

16. Sur cette question, $c f$. C. de France, Anthropologie et Cinéma, Paris, Éd. de la Maison des Sciences de l'Homme, (1982) 1989, p. 174.

17. C. Townsend-Gault, art. cit., p. 161.

18. Ibid., p. 163.

19. S'extasier, comme il nous est arrivé de l'entendre, devant les qualités esthétiques, formelles de Fontaine et s'en dire ému, tout en refusant de considérer ces mêmes qualités présentes dans un urinoir qui a conservé sa fonction, c'est faire découler l'expérience esthétique de l'attribution du terme «art» à un objet («Puisque c'est une œuvre d'art, l'objet doit nécessairement posséder des qualités esthétiques dont je dois faire l'expérience»). Or, comment un même objet, placé dans des contextes différents, pourrait-il posséder en propre certaines qualités qu'il ne posséderait pas dans l'autre contexte?

20. Le passage de la catégorie "document ethnographique » à la catégorie "art" ne change fondamentalement rien: dans les deux cas, l'objet est coupé de son contexte initial de fonctionnement et se voit attribuer de nouveaux rôles à jouer.

21. L. Shiner, " "Primitives Fakes", "Tourist Art", and the Ideology of Authenticity ", The Journal of Asthetics and Art Criticism, vol. 52, no 2, printemps 1994, p. 225-234; ici p. 226. 
22. F. Willett, "True or False : the False Dichotomy», African Art, vol. IX, no3, p. 8-14, cité par L. Stéphan, "Le vrai, l'authentique et le faux", Cahiers du Musée national d'art moderne, n³6, été 1991, p. 23.

23. En théorie, sinon en pratique: on sait toute la difficulté que peut poser l'authentification d'une œuvre, et les musées occidentaux ne sont sans doute pas exempts de faux au sens défini par F. Willett, ou au moins de pièces non absolument authentiques.

24. M. Nesbit, "Les originaux des ready-mades: le modèle de Duchamp ", Cahiers du Musée national d'art moderne, $\mathrm{n}^{\circ} 33$, automne 1990, p. 33-65; ici p. 62.

25. Il est un cas, cependant, où les masques sont employés comme matériaux, il s'agit des inclusions d'Arman. Mais l'artiste lui-même admet que les masques utilisés sont de moindre valeur et que, d'autre part, ils peuvent être récupérés.

26. M. Duchamp, Duchamp du signe, op. cit., p. 192.

27. Loin d'être anecdotique, cette "différence territoriale» entraîne une autre distinction entre ready-made et arts dits primitifs: les objets qui constituent les ready-made sont des objets familiers, identifiables, dont on connaît l'usage, bref qui font partie de l'environnement quotidien du spectateur. En revanche, les objets provenant des sociétés extra-occidentales n'appartiennent pas à ce quotidien; ils sont marqués par l'étrangeté, l'extranéité, l'exotisme. En d'autres termes, ils ne sont pas, en Occident, des objets usuels; ils sont avant tout des objets de collection.

28. T. de Duve affirme cependant que la pelle dont il se sert peut être considérée comme une réplique du ready-made de Duchamp : «La pelle à neige qui pend dans mon garage a droit tout autant qu'une autre au titre de réplique authentique d'un Duchamp, elle est aussi unique, ni plus ni moins, que chacun des huit tirages que leur auteur autorisa en cédant son copyright à Arturo Schwartz. Mais malgré son unicité et son authenticité, ma pelle à neige n'a pas d'aura, n'en déplaise à Benjamin qui ne pouvait pas prévoir, en 1930, que "le sens du semblable dans le monde" se verrait intensifié à tel point que, moyennant la reconnaissance de la priorité de la reproduction sur l'original, la perception parviendrait à rendre unique un objet standard. Et ma pelle à neige n'a pas de statut. Elle ne porte pas une étiquette invisible qui dirait "ceci est de l'art", elle n'est pas inscrite, aurait dit Duchamp, sinon à l'encre sympathique de ma propre sympathie. Elle n'est plus "de l'art", elle est une ceuvre». T. de Duve, op. cit., p. 54-55. N'est-ce pas oublier, cependant, le rôle de la signature dans l'accession d'un objet au statut d'œuvre d'art?

29. "Dans le cas d'un ready-made, qui est l'artiste? Certainement pas le producteur de l'objet, qui n'a créé qu'un objet utilitaire. L'artiste, c'est bien entendu Marcel Duchamp [...]. Par conséquent, s'il en allait de même pour notre masque africain, il faudrait affirmer que l'artiste, ce n'est certainement pas l'Africain qui a sculpté le masque, mais le conservateur qui a eu l'idée géniale de l'élever au rang d'œuvre d'art en le délivrant de son usage rituel pour l'exposer dans un musée des Beaux-Arts. Et il en va de même pour tous les autres objets qui seront exposés dans le musée en question [quai Branly]. Voilà qui suffit à élever le conservateur au plus haut rang de l'histoire de l'art. Le plus grand artiste de l'art africain, océanien, américain et arctique est un Français! Splendide résultat théorique dont on appréciera toute la saveur en songeant aux conséquences juridiques et financières qui pourraient en être tirées». A. Séguy-Duclot, «Art. Dépasser le nihilisme", Utopia 3. La Question de l'art au troisième millénaire, Actes du colloque international-Université Paris VIII, Université de Venise, sous la dir. de C. Giordano Bruni, Paris, GERMS, 2002, p. 141-151; ici p. 146. 30. Et, est-il besoin de le préciser, ces acteurs appartiennent à la sphère occidentale. $C f$. le cas que rapporte $S$. Price à propos de la manière dont l'exposition Angles on African Art (Center for African Art, 1987) a été mise en place: demandant à différents membres du monde de l'art d'effectuer un choix parmi une présélection d'artefacts, Susan Vogel contacte l'artiste Baoulé Kaoumé, mais ne lui soumet que des objets provenant de sa propre société, l'estimant incapable de porter un jugement cohérent sur des objets provenant d'autres sociétés. Un tel mépris est confondant: pourquoi en effet restreindre la portée du jugement de cet artiste aux objets de sa seule société alors que les Occidentaux sont considérés aptes à émettre un jugement sur des objets provenant de sphères culturelles qui leur sont probablement plus étrangères qu'à Kaoumé? (S. Price, Arts primitifs; regards civilisés, trad. de l'anglais par G. Lebant, Paris, École nationale supérieure des beaux-Arts, 1995/The University of Chicago Press, 1989).

31. On pourrait effectivement s'interroger sur les exemples choisis dans les deux articles qui nous ont servi de point de départ. Dans les deux cas, il s'agit d'objets relevant du religieux, du sacré, et non d'objets de la vie courante. Pour quelle raison? Une hypothèse serait de dire que la dimension esthétique et artistique accordée aux objets est fonction du degré de religiosité, de sacralité dont l'objet est investi (M. Leiris décrit notamment la manière dont certains objets sont "acquis" par la mission DakarDjibouti : lorsque les villageois refusent de céder un de leurs objets, il arrive que les membres de l'équipe reviennent la nuit pour voler l'objet en question: si les villageois ont refusé de le céder, c'est qu'il doit posséder une plus grande valeur; on a là un lien entre le secret et le sacré. Cf. L'Afrique fantôme, Paris, Gallimard, 1981). Même si tous les objets de toutes les catégories sont exposés, comme nous l'avons dit, sans aucune hiérarchie, il n'en demeure pas moins que tous ne peuvent être mis sur le même plan. Si on voulait donner une cohérence à la comparaison entre l'apparition des ready-made et l'entrée des arts dits primitifs dans le musée, il serait sans doute plus pertinent de partir de l'exemple d'objets d'usage courant, qui ne seraient investis d'aucune sacralité.

32. S. Price, "Art autre - Art nôtre", Cabiers du Musée national d'art moderne, n²8, été 1989 (numéro consacré à l'exposition Magiciens de la terre), p. 61-68, ici p. 64.

33. Ibid., p. 66. "Comme celui d'un chien, le pedigree d'une œuvre d'art est un arbre généalogique qui fournit à l'acquéreur potentiel une garantie quant à la valeur de son achat. Dans ce sens, on peut le rapprocher de l'acte de propriété d'un bâtiment historique ou des pages jaunissantes d'un volume rare: il précise dans quels cadres on va, rétrospectivement, se mouvoir en devenant acquéreur. Le pedigree d'une œuvre d'art donne la liste non seulement des propriétaires précédents mais aussi les expositions et les publications où elle a figuré, les ventes où elle a changé de mains et les prix obtenus lors de chaque transaction" (S. Price, Arts primitifs; regards civilisés, op. cit., p. 154).

34. On retrouve ce type de considérations dans l'article d'A. Danto, «Art et artefact en Afrique ", Après la fin de l'art, trad. de l'anglais par C. HarySchaeffer, Paris, Seuil, 1996 (Farrar, Strauss \& Giroux, 1992, paru précédemment dans Art/Artifact. Africain Art in Anthropology Collections, catalogue de l'exposition organisée par S. Vogel au Center for Africain Art, New York)

35. S. Price, art. cit., note 32, p. 66.

36. Ibid., p. 61. 